\section{FC4 THE ALTERNATIVE PATHWAY IS CRITICAL FOR PATHOGENIC COMPLEMENT ACTIVATION IN DIET -INDUCED AND ENDOTOXIN-INDUCED ATHEROSCLEROSIS IN LOW-DENSITY LIPOPROTEIN RECEPTOR-DEFICIENT MICE}

\author{
doi:10.1136/hrt.2010.205781.10
}

${ }^{1} \mathrm{~T}$ H Malik, ${ }^{1} \mathrm{~A}$ Cortini, ${ }^{1} \mathrm{D}$ Carassiti, ${ }^{2} \mathrm{~V}$ W Y Leung, ${ }^{1} \mathrm{M} \mathrm{J}$ Lewis, ${ }^{2} \mathrm{D} 0$ Haskard, ${ }^{1} \mathrm{M}$ Botto. ${ }^{1}$ Rheumatology Section, Imperial College, London, UK; ${ }^{2} B H F$ Cardiovascular Medicine Unit, Imperial College, London, UK

Our previous studies have shown that the early components of the classical complement pathway protect low-density lipoprotein receptor deficient mice ( $\mathrm{Ldl}-\mathrm{r}-\mathrm{-}$ ) from atherogenesis when fed a low-fat diet. However, the role of the alternative pathway remains unknown. To investigate this, we crossed mice lacking the alternative pathway activator Factor $\mathrm{B}(\mathrm{Bf}-/-)$ with Ldlr-/- mice. Lipid profiles after 12 weeks on a high-fat diet showed significantly reduced levels of total cholesterol $(25.2 \% \pm 0.9 \mathrm{mmol} / \mathrm{l}, \mathrm{n}=14$, vs $40.5 \% \pm 1.8 \mathrm{mmol} / \mathrm{l}, \mathrm{n}=17, \mathrm{p}<0.0001)$ and lipoproteins $(\sim 1.5$-fold lower VLDL and LDL) in the $\mathrm{Bf}-/-$.Ldlr $-/-$ mice compared with the Ldlr-/- animals. Consistent with this, high-fat fed Bf-/-. Ldlr-/ - mice had decreased cross-sectional aortic root lesion fraction area (median $12.2 \%$, IOR $8.92 \%$ to $15.82 \%$ vs $16.3 \%, 14.76 \%$ to $19.74 \%, p=0.0016)$ and reduced lesion complexity. These changes were associated with reduced complement activation in the circulation and in atherosclerotic plaques. There was no difference in lipid profiles between $\mathrm{Bf}-/$ - . Ldlr-/ - and Ldlr-/ - mice fed a lowfat diet, but in these groups administration of lipopolysaccharide (LPS) led to significant increase in atherosclerosis only in Ldlr-/and not in $\mathrm{Bf}-/-. \mathrm{Ldl}-/-$ (aortic root lesion fraction: $\mathrm{Bf}-/-$. Ldlr-/-: $7.85 \%$, range 4.93 to $15.24 \%, n=9$; $\mathrm{Ldlr}-/-: 22.53 \%$, range 17.36 to $25.25 \%, n=10, p=0.0009)$, indicating that the alternative pathway also has a key role in endotoxin-mediated exacerbation of disease. These data indicate that amplification of complement activation by the alternative pathway in response to diet or infection may convert the complement system from a protective to a more atherogenic role.

\section{FC5 REAL TIME IMAGING OF LYMPHOCYTES IN APOE-/- MOUSE AORTIC TERTIARY LYMPHOID ORGANS}

\section{doi:10.1136/hrt.2010.205781.11}

${ }^{1} \mathrm{G}$ Grassia, ${ }^{1} \mathrm{G}$ J Dever, ${ }^{1} \mathrm{P}$ Gordon, ${ }^{1} \mathrm{I}$ B McInnes, ${ }^{2} \mathrm{R}$ Spanbroek, ${ }^{2} \mathrm{~A}$ J R Habenicht, ${ }^{1} \mathrm{~J} \mathrm{M}$ Brewer, ${ }^{1} \mathrm{P}$ Garside, ${ }^{1} \mathrm{P}$ Maffia. ${ }^{1}$ Division of Immunology, Infection \& Inflammation, University of Glasgow, UK; ${ }^{2}$ Institute for Vascular Medicine, Friedrich Schiller University of Jena, Germany

Rationale for the study Recent developments in imaging technologies such as multiphoton laser-scanning microscopy (MPLSM) have allowed real time in vivo imaging and analysis of immune cells interactions in the context of the immune system, providing important insight into the dynamics of a developing immune response. However, a number of technical limitations have precluded real time imaging of individual immune cells within intact atherosclerotic vessels. Here we provide a feasible protocol for MPLSM with real time imaging and analysis, at a cellular resolution, of adoptively transferred lymphocytes, in the adventitia of intact ApoE-/- mouse arteries and the associated aortic tertiary lymphoid organs (ATLOs) which have recently been implicated in the late stages of atherosclerosis (JEM 2009;206:233-48).

Methodology Cell suspensions were prepared from peripheral and mesenteric lymph nodes of C57BL/6 mice and labelled with Cell TrackerTM Red CMTPX (Molecular Probes). Lymphocytes were injected IV into aged ApoE-/- (80-87 weeks) recipients. Twenty four hours after transfer, excised intact mouse abdominal aortas were imaged by MPLSM and analysed by Volocity 5 software (Improvision).
Results and conclusions The use of this system enabled real time imaging of immune cells in atherosclerotic vessels at a cellular resolution. At $24 \mathrm{~h}$ after transfer several infiltrated lymphocytes were detectable in the ATLO in aged ApoE-/- mice. Cells showed a great motility with a mean velocity of $0.45 \mu \mathrm{m} / \mathrm{s}$ and a pick velocity of $1.16 \mu \mathrm{m} / \mathrm{s}$. Consequently, this system could be a powerful tool to study the atherosclerotic immune responses.

Acknowledgements This work is supported by a BHF grant (PG/06/083/21198).

\section{BAS/BSCR poster abstract}

\begin{tabular}{|l|l}
\hline BAS/ & VACCINATION AGAINST INFLUENZA PROMOTES STABLE \\
BSCR1 & ATHEROSCLEROTIC PLAQUES IN APOE - /- MICE
\end{tabular}

doi:10.1136/hrt.2010.205781.12

A Bermudez-Fajardo, E Oviedo-Orta. Faculty of Health and Medical Sciences, University of Surrey, Guildford, Surrey, UK

Current evidence suggests a relationship between seasonal influenza viral infection and cardiovascular disease (CVD) complications. Experimentally, animals inoculated with influenza A virus develop thrombotic complications similar to those seen in humans. Conversely, several clinical trials have suggested that influenza vaccination may have a protective effect on CVD. However, the potential mechanisms remain unstudied. In order to study this, we vaccinated six groups of male ApoE-/- mice with 0.36, 1.8, 9 and $45 \mu \mathrm{g} / \mathrm{ml}$ of Vaxigrip (influenza vaccine, Sanofi Aventis), $45 \mu \mathrm{g} / \mathrm{ml}$ of Pneumo23 (Pneumococcus vaccine, Sanofi Aventis) and phosphatebuffered saline (PBS). Animals received one injection and two boosters 3 weeks apart before starting on a high-fat diet for 12 weeks. Plasma was collected pre-immunisation and at termination for profile analysis of antibodies, lipids and cytokines. Analysis of atherosclerosis development was carried out using paraffin sections of the brachiocephalic artery. We observed an increase in anti-influenza IgG1 but not in IgG2a, IgG2b, IgA or IgM in any of the groups. Animals that received Pneumo23 vaccine only showed increased levels of vaccine-specific IgM. No significant changes in plasma levels of lipids were noted. Animals vaccinated with $45 \mu \mathrm{g} /$ $\mathrm{ml}$ Vaxigrip developed smaller atherosclerotic lesions with lower lipid content but were richer in inflammatory cells and collagen than control animals. Other groups showed no significant differences in plaque area, lumen and tunica media area. Animals vaccinated with $45 \mu \mathrm{g} / \mathrm{ml}$ Vaxigrip showed lower levels of interleukin (IL) 2, interferon $\gamma$ and IL-17 and higher levels of IL-4. Our results suggest that vaccination against influenza may protect against CVD by inducing stable and smaller atherosclerotic plaques.

\section{BAS/ ANALYSIS OF MICROVESSELS WITHIN CAROTID PLAQUES BSCR2 COMPARISON OF SYMPTOMATIC AND ASYMPTOMATIC PATIENTS TO IDENTIFY THOSE AT RISK FROM PLAQUE RUPTURE}

doi:10.1136/hrt.2010.205781.13

${ }^{1} \mathrm{E} J$ R Hill, ${ }^{1} \mathrm{M}$ Chowdhury, ${ }^{2} \mathrm{~F}$ Serracino-Inglott, ${ }^{2} \mathrm{~J} V$ Smyth, ${ }^{3} \mathrm{M}$ Slevin, ${ }^{1} \mathrm{M}$ Y Alexander. ${ }^{1}$ Cardiovascular Research Group, The University of Manchester, Core Technology Facility, UK; ${ }^{2}$ Department of Vascular Surgery, Manchester Royal Infirmary, UK; ${ }^{3}$ School of Biology Chemistry and Health Science, Manchester Metropolitan University, UK

Introduction Stroke is the third most common cause of death world wide. Most cases of stroke are caused by atherosclerotic plaque rupture. A challenge facing clinicians is identifying asymptomatic patients at risk of plaque instability. Locally released angiogenic growth factors may contribute to microvessel instability within 